\title{
Baby blues na atenção primaria: reflexos entre mãe e o recém-nascido
}

\author{
Baby blues in primary attention: reflections between mother and the newborn \\ Baby blues en atención primaria: reflexiones entre la madre y el recién nacido \\ Elessandra Ramos Pedrosa ${ }^{1}$, Michelli Domingos da Silva ${ }^{2 *}$, Railton da Silva Miranda².
}

\begin{abstract}
RESUMO
Objetivo: Descrever os efeitos da Baby blues pós-parto, na interação entre mãe e bebê. Métodos: Trata-se de uma pesquisa, de revisão integrativa, de dados secundários, do tipo observacional. Os dados coletados são originários de artigos científicos, revistas cientificas da área da enfermagem como: Primeiramente, na Biblioteca Virtual de Saúde (BVS), Medical Literature Analysis and Retrieval System Online (MEDLINE), Scientific Eletronic Library Online (SciELO), Literatura Latino-Americana e do Caribe em Ciências da Saúde (LILACS). Resultados: A depressão e a ansiedade materna refletem negativamente no desenvolvimento do bebê e são capazes de acarretar danos nas relações pessoais que esta possa estabelecer ao longo da sua vida. Entretanto os resultados podem aparecer como danos no desenvolvimento infantil, no processo da amamentação, comunicação verbal do bebê e assim por diante. Conclusão: De acordo com o referencial bibliográfico revisado, foi possível verificar como os impactos do baby blues podem influenciar durante a gestação e no pós-parto.
\end{abstract}

Palavras-chave: Depressão, Enfermagem, Pré-Natal.

\section{ABSTRACT}

Objective: Describe the effects of postpartum Baby blues on the interaction between mother and baby. Methods: It is a research, integrative review, secondary data, observational type. The data collected comes from scientific articles, scientific journals in the field of nursing, such as: First, in the Virtual Health Library (VHL), International Literature in Health Sciences (MEDLINE), Scientiic Eletronic Library Online (SciELO) and Latin American and Caribbean Literature in Health Sciences (LILACS). Results: Maternal depression and anxiety reflect negatively on the baby's development and are capable of causing damage to the personal relationships that the latter may establish throughout his life. However, the results can occur as damage to child development, the breastfeeding process, verbal communication of the baby, and so on. Conclusion: According to the revised bibliographic reference, it was possible to verify how the impacts of the baby blues can influence during pregnancy and postpartum.

Keywords: Depression, Nursing, Prenatal.

\section{RESUMEN}

Objetivo: Describir, a través de una revisión de la literatura, los efectos del Baby blues posparto en la Objetivo: Describa los efectos. Baby blues posparto, en la interacción entre madre y bebé. Métodos: Es una investigación, revisión integradora, datos secundarios, tipo observacional. Los datos recopilados provienen de artículos científicos, revistas científicas en el campo de la enfermería, tales como: Primero, en la Biblioteca Virtual en Salud (BVS), Literatura Internacional en Ciencias de la Salud (MEDLINE), Biblioteca científica electrónica en línea (SciELO) y Literatura Latinoamericana y del Caribe en Ciencias de la Salud (LILACS). Resultados: La depresión y la ansiedad maternas se reflejan negativamente en el desarrollo del bebé y son capaces de dañar las relaciones personales que este último puede establecer a lo largo de su vida. Si embargo los resultados pueden ocurrir como daños al desarrollo infantil, el proceso de lactancia, la comunicación verbal del bebé, etc. Conclusión: De acuerdo con la referencia bibliográfica revisada, fue posible verificar cómo los impactos de la melancolía pueden influir durante el embarazo y el posparto y así sucesivamente.

Palabras-clave: Depresión, Enfermería, Cuidados prenatales.

1 Universidade Nilton Lins (UNL), Manaus - AM. *E-mail: michelliclarinha@hotmail.com 


\section{INTRODUÇÃO}

A depressão pós-parto vem sendo um motivo de grande preocupação por estudiosos e especialistas, visando o grande aumento da prevalência desta problemática, ressaltando assim que o dano causado à mãe interfere no diagnóstico, como pelo seu conflito no desenvolvimento infantil. Essa agregação de fatores coloca em questionamento a amplitude de um problema de saúde pública que, precisa de apoio e atenção por partes das autoridades, pesquisadores e profissionais da área de saúde mental (MARTINEZ P, et al., 2016; MORAES GPA, et al., 2017).

Segundo Ramos ASMB, et al., (2018), sendo o parto considerado um fenômeno natural, a mulher deve ser assistência da por profissionais multidisciplinar, com o intuito de minimizar as irregularidades durante as consultas de pré-natal, trabalho de parto, parto e pós-parto. Pois uma boa assistência e um bom atendimento pode diminuir o surgimento de problemas reais facilitando a identificação diagnósticos, planejamentos e implementação de cuidados necessários.

A depressão pós-parto (DPP) é definida como uma doença neurológica, com episódio que ocorreram maiores que ocorrem nas primeiras quatro semanas pós-parto. No entanto, o quadro clínico da depressão pós-parto é bastante heterogêneo, sendo que sintomas de ansiedade são mais comuns nesta ocasião do que em outros períodos da vida.

Além disso, não é incomum descobrir sintomas de obsessão e compulsão na puérpera, até mesmo com pensamentos de causar injúrias ao bebê, o que deve ser diferenciado da psicose pós-parto. Blues pós-parto é uma transitória caracterizada por alteração do humor, de leve a moderada intensidade, frequentemente rápida, que envolve sensação de tristeza, irritabilidade, ansiedade, redução da concentração, insônia, choro fácil e crises de choro. Alguns artigos científicos descrevem que $40 \%$ a $80 \%$ das puérperas desenvolvem tais alterações de humor, isso pode acontecer entre duas a três semanas após o parto.

Quanto à prevalência da DPP, encontramos uma grande variação que ocorre em função da falta de igualdade nos parâmetros metodológicos que vem sendo utilizados nas pesquisas, tais como, alterações na diferenciação da amostra, nas ferramentas de rastreios empregados e no período de realização do mesmo( MORAIS MLS, et al., 2015).

Pesquisas revelam que o comportamento de mães deprimidas pode afetar o desenvolvimento de psicopatologias em seus filhos, ou seja, a DPP pode levar a ocorrência de desordens comportamentais, afetivas, cognitivas e sociais, autoimagem negativa, distúrbios do apego e maior incidência de diagnóstico psiquiátrico. Alguns autores comentam que a puérpera ao perceber que seu tempo ficou limitado e direcionado aos cuidados do bebê, a preocupação e o desespero surgem de como retornar a sua atividade profissional. Excepcionalmente algumas mães abrem mão da carreira profissional para se dedicar à maternidade, contribuindo então para que se sinta triste e incapaz, tornando-a muitas das vezes financeiramente dependente (GREINERT BRM e MILANI RG, 2015).

Esta breve descrição reflete a falta de concordância nos manuais oficiais que norteiam os profissionais e pesquisadores da área. $O$ esperado seria que tais manuais de classificações dos transtornos mentais apresentassem um padrão e refletisse não apenas nos avanços científicos no conhecimento de causas e consequência dos diagnósticos, mas também seu uso comum na prática dos profissionais de saúde (FRANCES A, 2015).

De acordo com Moraes GPA, et al., (2017), estudos descrevem sobre a necessidade de se realizar uma busca aprofundada sobre o diagnóstico. Neste período de seis meses para a ocorrência do diagnóstico se faz necessário a realização de alguns exames para avaliar a prevalência de depressão, ansiedade e transtorno de estresse pós-traumático entre mães durante o período perinatal, examinando os percursos desses transtornos da gravidez até seis meses pós-parto.

Desta forma, é necessário ter um profissional de enfermagem nas consultas de pré-natal, sendo necessário maiores discussões sobre a temática, que estimulem a busca de estratégias para enfrentamento desse problema. O objetivo desse estudo é descrever os efeitos da Baby blues pós-parto, na interação entre mãe e bebê. 


\section{MÉTODOS}

Trata-se de uma pesquisa, de revisão integrativa, de dados secundários, do tipo observacional (MARCONI MA e LAKATOS EV, 2017). Os dados coletados são originários de artigos científicos, revistas cientificas da área da enfermagem como: Primeiramente, na BVS; MEDLINE, SciELO, LILACS. A BVS, desenvolvida sob coordenação do BIREME, é uma rede de fontes de informação online para a distribuição de conhecimento científico e técnico em saúde. De acordo com as seguintes palavras chaves: Depressão; Enfermagem; PréNatal.

Foram utilizados os critérios de inclusão da pesquisa foram: artigos que contemplem a temática de acordo com os descritores pesquisados, artigos no idioma português e inglês, artigos que possuam título, local de pesquisa, editora, ano de publicação. Como critérios de exclusão da pesquisa retiramos: pesquisas não originais, pesquisas não completas, com apenas o resumo ou faltando a parte de resultados e discussão. Pesquisas de Teses, dissertações, revisões narrativas e relatos de experiência.

Foram pesquisados os seguintes dados: Título do artigo, título do periódico, autores idiomas, ano de publicação, instituição sede do estudo, tipo de publicação, objetivo ou questão de investigação, amostra, tratamento de dados, investigação, nível de evidências. Para a adequação da pesquisa, segue-se então a categorização por meio da tabela mundialmente conhecida de Oxford para delineamento de evidências cientificas Figura 1.

Figura 1 - Fluxograma representativo a respeito dos critérios de inclusão e exclusão.

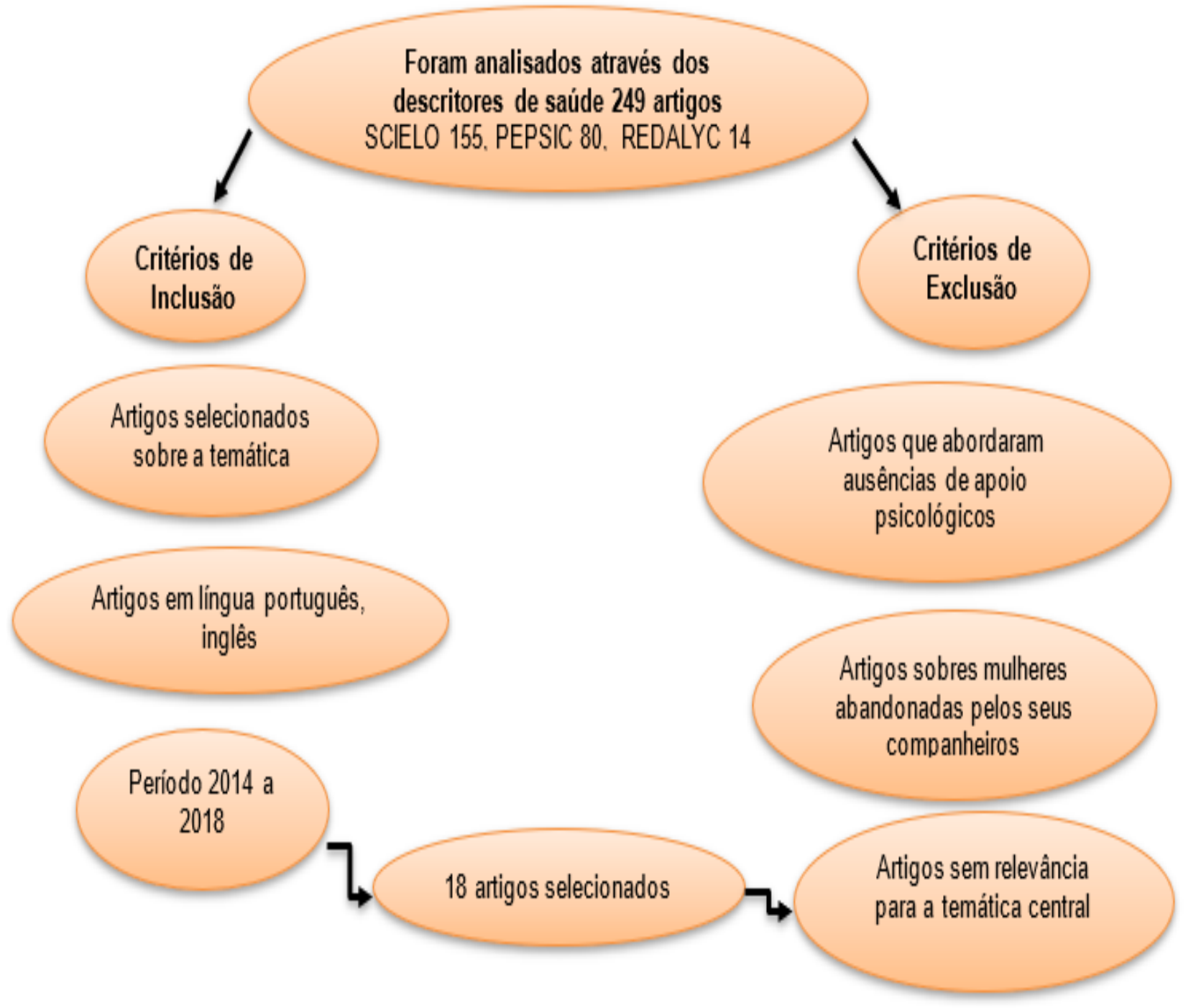

Fonte: Pedrosa ER, et al., 2020. 
As informações coletadas e analisadas foram para o leitor, melhor interpretar e adquirir conhecimento e informação sobre dimensionamento de pessoal na enfermagem e evidenciar as mudanças ocorridas. Para facilitar a instrumentalização, os procedimentos foram realizados em fases:

$1^{\text {a }}$ Fase - Nela realiza- se a criação das perguntas norteadoras: determinando quais estudos devem ser incluídos quais os meios adotados e que informações serão coletadas;

$2^{\underline{a}}$ Fase - Início da busca nas bases de dados eletrônicas por meio da SCIELO / PEPSICO / REDALYC / OMS / MS, em consonância com os critérios de inclusão e exclusão a fim de garantir a representatividade das informações coletadas;

$3^{\text {a }}$ Fase - Tratamento dos dados obtidos: onde deve- se resumir e organizar os achados através de um instrumento, para assegurar que a totalidade de artigos relevantes seja extraída, minimizar o risco de erros na transcrição, garantir precisão na checagem das informações e servir como registro;

4a Fase - Nesta, organiza-se a análise crítica dos estudos: sendo necessário organizar a abordagem que será utilizada delinear o rigor e as características de cada estudo, utilizando a PBE para classificá-los em níveis de evidências;

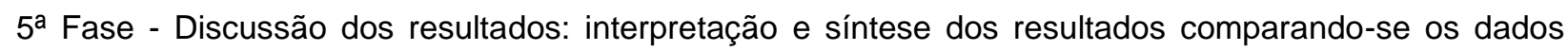
evidenciados na análise dos artigos ao referencial teórico e identifica possíveis lacunas de conhecimento. $O$ pesquisador deve salientar suas conclusões e inferências, bem como explicitar vieses;

$6^{\underline{a}}$ Fase - A etapa final é a de apresentação de revisão integrativa: que deve incluir informações pertinentes oportunizando ao leitor a avaliar os procedimentos empregados na elaboração da revisão, seus aspectos que relacionem os tópicos abordados e o detalhamento dos estudos incluídos;

O estudo tratou-se de pesquisar através de dados secundários, respeitando a citação dos autores de acordo com as normas ABNT (NBR6023), não há necessidade de aprovação em Comitê de Ética em Pesquisa e nem de termos de consentimento, tendo em vista que todas as informações estão disponíveis gratuitamente em domínio público e atendem as normas da Resolução 466/12 do Conselho Nacional de Saúde.

\section{RESULTADOS E DISCUSSÃO}

Posterior à análise criteriosa dos estudos compilados nas bases de dados através do uso de palavraschaves, obteve-se os resultados a seguir: um universo de 249 artigos divididos entre as bases de dados; SCIELO 155, PEPSIC 80 e REDALYC 14.

Após a aplicação dos critérios de seleção, restaram 18. Após a divisão e aplicação dos critérios de inclusão e exclusão dos artigos, criou-se um quadro de resultados que possibilitou a identificação dos estudos selecionados segundo: o ano de publicação, título dos periódicos/classificação, país de origem/idioma, base de dados e nível de evidência (Quadro 1).

De acordo com os artigos selecionados através dos descritores: Depressão Pós-parto, Enfermagem, PréNatal. Os dados obtidos através dos artigos demonstraram que com relação à temática que vêm sendo construída de forma crescente, os profissionais de saúde estão compreendendo o seu papel fundamental na reversão dos índices da DPP, visto que apesar de todas as evidências científicas é preciso compreender como ocorre esse processo. Portanto, isso demonstra uma necessidade impreterível de se pesquisar, pois, apesar de estar vulnerável ao desenvolvimento de transtornos psíquicos poderá contribuir no suporte à mãe deprimida e ao bebê, com relação a isso, os estados demostram que tem sido pouco investigado.

O desenvolvimento de competências profissionais é ocasionada pela vivência, e destacada como uma ferramenta para o profissional especializado. Essa afirmação parte das pesquisas realizadas de acordo com os artigos acima, aonde a maioria são enfermeiros, psicólogos e médicos experientes. A prática na formação profissional de saúde também apresenta o seu valor, visto grande parte dos artigos que integram o grupo com experiência (Quadro 2). 
Quadro 1 - Distribuição dos estudos selecionados por ano de publicação, base de dados, idioma, título do estudo, quales capes, país de publicação.

\begin{tabular}{|c|c|c|c|c|c|c|}
\hline $\begin{array}{l}\text { № do } \\
\text { artigo }\end{array}$ & $\begin{array}{c}\text { Ano de } \\
\text { publicação }\end{array}$ & $\begin{array}{l}\text { Base de } \\
\text { dados }\end{array}$ & Idioma & Título do estudo & $\begin{array}{l}\text { Quales } \\
\text { capes }\end{array}$ & $\begin{array}{c}\text { País de } \\
\text { publicação }\end{array}$ \\
\hline 1 & 2017 & Scielo & Português, Inglês, Espanhol & Tristeza materna em puérperas e fatores associados & $\mathrm{B} 2$ & Brasil \\
\hline 2 & 2014 & Scielo & Português e inglês & $\begin{array}{c}\text { Development of Children's Personality: The role of early childhood } \\
\text { education. }\end{array}$ & B2 & Brasil \\
\hline 3 & 2015 & Pepsic & Português & $\begin{array}{l}\text { Depressão pós-parto materna: crenças, práticas de cuidado e estimulação } \\
\text { de bebês no primeiro ano de vida }\end{array}$ & B3 & Brasil \\
\hline 4 & 2015 & Scielo & Português & $\begin{array}{c}\begin{array}{c}\text { Bebês com Sequência de Pierre Robin: saúde mental materna e interação } \\
\text { mãe-bebê }\end{array} \\
\end{array}$ & A1 & Brasil \\
\hline 5 & 2017 & Scielo & Português e espanhol & Depressão pós-parto: uma revisão sobre fatores de risco e de proteção & B2 & Brasil \\
\hline 6 & 2014 & Redalyc & Português & $\begin{array}{l}\text { Alojamento conjunto em um hospital universitário: depressão pós-parto na } \\
\text { perspectiva do enfermeiro }\end{array}$ & B2 & Brasil \\
\hline 7 & 2018 & Scielo & Português e espanhol & $\begin{array}{l}\text { Prevalence of depression and associated factors in women covered by } \\
\text { Family Health Strategy }\end{array}$ & B 3 & Brasil \\
\hline 8 & 2015 & Scielo & Português e espanhol & $\begin{array}{c}\text { Legalização da Prescrição de Medicamentos Pelo Enfermeiro no Brasil: } \\
\text { História, Tendências e Desafios }\end{array}$ & $\mathrm{A} 2$ & Brasil \\
\hline 9 & 2015 & Scielo & Português e inglês & $\begin{array}{l}\text { Challenges for primary healthcare professionals in caring for women with } \\
\text { postpartum depression }\end{array}$ & A 2 & Brasil \\
\hline 10 & 2018 & Scielo & Português e inglês & $\begin{array}{c}\text { Sintomas depressivos em puérperas atendidas em unidades de saúde da } \\
\text { família }\end{array}$ & B1 & Brasil \\
\hline 11 & 2017 & Scielo & Português e inglês & $\begin{array}{c}\text { Planejamento familiar e papéis parentais: o tradicional, a mudança e os } \\
\text { novos desafios }\end{array}$ & B1 & Brasil \\
\hline 12 & 2015 & Scielo & Português & $\begin{array}{c}\text { Concepções sobre desenvolvimento infantil na perspectiva de educadoras } \\
\text { em creches públicas e particulares }\end{array}$ & B4 & Brasil \\
\hline 13 & 2014 & Scielo & Inglês e espanhol & $\begin{array}{l}\text { Saúde mental, interação mãe-criança e desenvolvimento ao final do } \\
\text { primeiro ano de vida. }\end{array}$ & A1 & Brasil \\
\hline 14 & 2017 & Redalyc & Português e Inglês & $\begin{array}{l}\text { A relação mãe-bebê na presença e na ausência de sintoma psicofuncional } \\
\text { no bebê: um estudo comparativo }\end{array}$ & B2 & Brasil \\
\hline 15 & 2014 & Scielo & Inglês e espanhol & $\begin{array}{l}\text { Saúde mental, interação mãe-criança e desenvolvimento ao final do } \\
\text { primeiro ano de vida }\end{array}$ & A1 & Brasil \\
\hline 16 & 2015 & Scielo & Português e inglês & $\begin{array}{c}\text { The Understanding Of The Nurse Facing The Identification Of Postpartum } \\
\text { Depression }\end{array}$ & $\mathrm{A} 2$ & Brasil \\
\hline 17 & 2016 & Pepsic & Português e inglês & A Família e o Desenvolvimento Infantil sob a Ótica da Gestalt-Terapia & B4 & Brasil \\
\hline 18 & 2017 & Scielo & Inglês & $\begin{array}{l}\text { Triagem e diagnóstico da depressão pós-parto: quando e } \\
\text { como? Tendências em psiquiatria e psicoterapia }\end{array}$ & B3 & Brasil \\
\hline
\end{tabular}

Fonte: Pedrosa ER, et al., 2020. 
Quadro 2 - Artigos evidenciados de acordo com a titulação profissional dos autores.

\begin{tabular}{|c|c|c|c|c|c|}
\hline $\begin{array}{l}\text { № do } \\
\text { artigo }\end{array}$ & Nome da revista & $\begin{array}{c}\text { Nível de } \\
\text { evidência }\end{array}$ & $\begin{array}{l}\text { Titulação dos } \\
\text { autores }\end{array}$ & Profissão & $\begin{array}{l}\text { Estado de } \\
\text { origens dos } \\
\text { artigos }\end{array}$ \\
\hline 1 & $\begin{array}{c}\text { Revista Portuguesa de } \\
\text { Enfermagem De Saúde } \\
\text { Mentad }\end{array}$ & $2 \mathrm{~B}$ & Doutora & Enfermeira & Paraná \\
\hline 2 & Psicologia em Estudo & $2 \mathrm{C}$ & Doutora & Pedagoga & Amazonas \\
\hline 3 & Psico & $3 A$ & Mestre & Psicóloga & São Paulo \\
\hline 4 & Estud. psicol. (Campinas), & $2 \mathrm{~B}$ & Doutora & Psicóloga & São Paulo \\
\hline 5 & $\begin{array}{l}\text { Psicologia: Ciência e } \\
\text { Profissão }\end{array}$ & $2 B$ & Doutora & Psicóloga & São Paulo \\
\hline 6 & $\begin{array}{c}\text { Revista de Pesquisa: } \\
\text { Cuidado e Fundamental } \\
\text { (Online) }\end{array}$ & $2 \mathrm{~B}$ & Doutor & Enfermeira & Rio de Janeiro \\
\hline 7 & $\begin{array}{l}\text { Jornal Brasileiro de } \\
\text { Psiquiatria }\end{array}$ & $2 B$ & $\mathrm{PhD}$ & Médicos & Minas Gerais \\
\hline 8 & $\begin{array}{c}\text { Legalização da Prescrição de } \\
\text { Medicamentos Pelo } \\
\text { Enfermeiro no Brasil: } \\
\text { História, Tendências e } \\
\text { Desafios }\end{array}$ & $2 \mathrm{~B}$ & Doutor & Enfermeiro & Bahia \\
\hline 9 & $\begin{array}{l}\text { Texto \& Contexto- } \\
\text { Enfermagem }\end{array}$ & $2 B$ & $\mathrm{PhD}$ & Enfermeira & Paraíba \\
\hline 10 & $\begin{array}{c}\text { Revista Brasileira de Saúde } \\
\text { Materno Infantil }\end{array}$ & $2 \mathrm{~B}$ & Doutora & Enfermeira & Pernambuco \\
\hline 11 & Cad. Saúde Pública & $2 B$ & Mestre & Enfermeira & $\begin{array}{c}\text { Rio Grande do } \\
\text { Sul }\end{array}$ \\
\hline 12 & $\begin{array}{c}\text { Revista Portuguesa de } \\
\text { Educação }\end{array}$ & $3 A$ & Doutora & Psicóloga & Paraíba \\
\hline 13 & Paidéia (Ribeirão Preto) & $2 \mathrm{~B}$ & Docente & Psicóloga & Rio de Janeiro \\
\hline 14 & $\begin{array}{c}\text { Boletim Academia Paulista } \\
\text { de Psicologia }\end{array}$ & $3 A$ & Doutora & Psicóloga & São Paulo \\
\hline 15 & Paidéia (Ribeirão Preto) & $2 \mathrm{~B}$ & Doutora & Psicóloga & São Paulo \\
\hline 16 & $\begin{array}{l}\text { Texto \& Contexto- } \\
\text { Enfermagem }\end{array}$ & $2 B$ & Doutora & $\begin{array}{l}\text { Enfermeira e } \\
\text { Docente }\end{array}$ & Paraíba \\
\hline 17 & $\begin{array}{l}\text { Periódicos eletrônicos em } \\
\text { psicologia }\end{array}$ & $2 A$ & Doutora & $\begin{array}{l}\text { Psicóloga e } \\
\text { Docente }\end{array}$ & Minas gerais \\
\hline 18 & $\begin{array}{c}\text { Tendências Psychiatry } \\
\text { Psychother. }\end{array}$ & $1 \mathrm{~B}$ & Especialista & Enfermeira & $\begin{array}{c}\text { Rio Grande do } \\
\text { Sul }\end{array}$ \\
\hline
\end{tabular}

Fonte: Pedrosa ER, et al., 2020.

Oliveira MJM e Dunningham WA (2015), comentam que os profissionais de saúde devem procurar estabelecer com o indivíduo um relacionamento de confiança e conduta, prestar cuidados específicos, caso esteja deprimida. Se houver necessidade estimular e oferecer atividades construtivas; observar e ouvir atentamente indícios de ideia suicida e estimular os cuidados pessoais como higiene, vestuário e alimentação.

Costa TE, et al. (2016), relatam que a DPP é o mal da existência humana, sua incidência varia de $10 \%$ a $20 \%$, configurando como sério problema de saúde materna, pois provoca diferentes alterações emocionais e comportamentais na mãe depressiva, e pode atingir de 10 a 15\% de mulheres, após o nascimento do filho, exigindo tratamento adequado. Já Oliveira CC, et al. (2019), relata que a DPP de modo geral, representa o 
mesmo quadro clínico típico da depressão em outras ocasiões da vida feminina, sucedido de particularidades relativas à maternidade em si e ao desempenho do papel de mãe. Emoções negativas, desinteresse pelo bebê e culpabilidade por não conseguir cuidar são frequentes e podem resultar em um desenvolvimento insatisfatório da interação mãe-bebê.

Para Meira BM, et al. (2015), a Organização Mundial de Saúde (OMS) relata que, 50\% dos casos de DPP são diagnosticados e, dentre esses, apenas $25 \%$ recebem os cuidados adequados. Deste modo, o profissional de enfermagem pode contribuir, significativamente, para a detecção precoce deste distúrbio, por meio da interação profissional-paciente, realizando o acolhimento, anamnese, observando o comportamento desta em relação ao recém-nascido e às pessoas com quem convive, e investigando a trajetória e possíveis causas que possam levá-la à depressão.

Para Oliveira CC, et al. (2019), se faz necessário a visita domiciliar da equipe de saúde, já que esta é fundamental para entender melhor como é a vida daquela gestante notando algum tipo de alteração e comunicando a equipe. Assim como se conhece uma determinada área/comunidade, é possível ter base para ações adequadas para cada mãe, promovendo proteção, prevenção, recuperação e reabilitação.

Segundo Hartmann JM, et al. (2017), esses fatores associados, a presença de sintomas depressivos, a história pregressa de saúde da paciente com relação a depressão ou doença mental, gravidez inesperada ou não aceita, ausência de parceiro ou de suporte social, alto nível de estresse percebido e ter sofrido eventos adversos na vida, história de abuso ou violência doméstica, história passada ou presente de complicações gestacionais e perda fetal. Esses fatores associados causam sequelas psicológicas até à própria morte dessa puérpera.

Já Guimarães RM, et al. (2017), relata que a gestação inesperada e a violência psicológica são fatores de risco para DPP para o começo de sintomas depressivos ao longo da gestação, tendo o parceiro e familiares como os principais agressores. A gestante molestada desenvolve um quadro de estresse emocional constante que se associa com baixa estima, isolamento e suicídio, uso excessivo ou abusivo de cigarro, álcool e drogas. Para Cardillo VA, et al. (2016), a DPP não está relacionada a ter ou não um companheiro, evidenciou-se que ser mãe solteira ou ter um baixo suporte familiar ou do pai da criança aumenta os riscos de mulheres desencadearem sentimentos negativos em relação à gravidez.

De acordo com Fonseca A e Canavarro MC (2017), o nível socioeconómico baixo (abrangendo o baixo aproveitamento da situação profissional da mãe) estabelece um fator de risco para a DPP, embora a magnitude do seu efeito seja baixa. Apesar de algumas investigações apontarem a idade materna (mulheres mais novas ou mulheres mais velhas) e o nível educacional (habilitações literárias mais baixas) como fatores de risco para a DPP, outras não reportam essa associação. O estado civil (ser solteira) também tem sido considerado um fator de risco para a DPP, apesar de os resultados não serem consistentes.

Para Costa TE, et al. (2016), a DPP é condicionada a ansiedade durante a gestação/puerpério ambas podem estão associadas à ocorrência de sintomatologia depressiva no período pós-parto, tendo esta associação uma magnitude elevada. Apesar de alguns estudos terem identificado que a história familiar de depressão pode constituir-se como um fator de risco para a DPP outras investigações não encontraram essa associação. Além disso, também existem alguns estudos que mostram a associação entre a melancolia, pósparto nomeadamente a intensidade dessa sintomatologia, e o posterior desenvolvimento de DPP.

Para Lima SS, et al. (2017), comenta que a identificação precoce dos sintomas depressivos na gestação, fornece subsídios para avaliação de risco e necessidade de encaminhamento, propiciando intervenções oportunas e desfechos materno-infantil mais favoráveis. A busca espontânea das gestantes por ajuda pode ser dificultada por alguns fatores como incapacidade de verbalizar sua necessidade de cuidado especializado comprometida pela presença dos sintomas depressivos, bem como não se sentir confiante para expor suas queixas aos profissionais uma vez que não é raro ouvirem que são sintomas comuns da gravidez.

Segundo a Portaria № 1.625 de 10 de julho de 2007, são de incumbência do enfermeiro das Estratégia Saúde da Família (ESF) realizar consultas de enfermagem, solicitar exames complementares e prescrever medicações, ressalvando as disposições legais da profissão e conforme os protocolos ou outras normas técnicas estabelecidas pelo Ministério da Saúde (MS) (MARTINIANO CS, et al., 2015). 
Para Andrade M, et al. (2017), é de suma importância que a consulta de enfermagem tenha como finalidade uma visão holística, detendo todo o conhecimento levado pelo paciente, possibilitando um diagnóstico preciso e apresentando condições de elaborar um plano de assistência de acordo com a necessidade de cada indivíduo para obtenção de um bom resultado.

Meira BM, et al. (2015), afirmaram que a atuação preventiva das equipes multidisciplinares durante a gestação pode proporcionar a nova mãe o apoio de que precisa para enfrentar os eventuais episódios de depressão. Entretanto, é importante admitir que a gestante expresse seus temores, queixas e ansiedades, podendo o profissional de enfermagem proporcionar assistência e orientação durante o acompanhamento do pré-natal, pois o atendimento precoce representa prevenção, ao qual traz grandes repercussões futuras.

Mozzaquatro O e Arpini DM (2017), O vínculo e a confiança entre a equipe multidisciplinar e a gestante que aconteça assim que ela chegar à unidade de saúde onde a mesma será recebida na para a realização de consultas pré-natais. Neste sentido Andrade M, et al., (2017), explana que a tecnologia e os melhorias científicas no contexto da gestação tem proporcionado aos profissionais da saúde o atendimento mais humanizado a mulher.

Para Pessoa L (2014), os profissionais de saúde estão habilitados para o acolhimento à está mulher em todo o ciclo de sua vida, proporcionando a promoção, prevenção de agravos e recuperação da saúde. As equipes de saúde devem ter conhecimentos diversos para atuar em várias áreas da saúde, considerando o indivíduo como um todo. Silva TR e Gontijo CS (2016), citou que todas as equipes de enfermagem devem estabelecer com a gestante um relacionamento de confiança, e observar os seus procedimentos, prestar cuidados específicos, caso ela esteja deprimida; estimular; oferecer atividades construtivas; observar e ouvir atentamente indícios de ideia suicida; e estimular os cuidados pessoais como higiene, vestuário e alimentação.

Os autores Hartmann JM, et al. (2017), explanam que as reuniões de grupos com gestantes, realizadas por profissionais da saúde, são indispensáveis no período do pré-natal, assim como elas comentam e compartilham suas vivências, com o intuito de aliviar os sentimentos de culpa, diminuir a insegurança, o sentimento de inferioridade e as expectativas sobre o bebê, ajudando na compreensão sobre o período que está passando, esclarecendo todas suas dúvidas.

Freitas DR, et al. (2014), Entretanto, a equipe multidisciplinar deverá realizar o monitoramento de todo o período de gestação focado no acolhimento mais humanizado, pois, através do mesmo ocorrerá um maior vínculo na relação mãe e filho, contribuindo, e ajudará na prevenção, no diagnóstico, e no tratamento da depressão pós-parto. Alguns autores advertem que é preciso conhecer as necessidades da puérpera, seus planos e projetos e compreender a singularidade de cada pessoa.

Silva TR e Gontijo CS (2016), relatam que os enfermeiros ao perceberem qualquer alteração psíquica da gestante, devem colocar a família ciente do que está ocorrendo. Advertem, ainda, que o trabalho em equipe multidisciplinar da saúde pode contribuir para que a gestante se sinta acolhida e confiante para expressar seus sentimentos. Igualmente, proporcionarão uma melhor superação das dificuldades da depressão pósparto tanto pela paciente, quanto pelos familiares.

A equipe de enfermagem é o facilitador e colaborador para que a mulher desenvolva habilidades para desempenhar o autocuidado e transformar a sua condição de saúde. A intervenção pode ser acondicionada a partir de consultas de enfermagem, visitas domiciliares, grupos educativos e ações na comunidade (FREITAS DR, et al., 2014).

Souza JM e Veríssimo MÓ (2015), relata que as puérperas sejam acompanhadas pela ESF e que os profissionais de saúde, estejam atentas as suas mudanças comportamentais, pois é fundamental para que a interação seja mais eficaz na tentativa de buscar algum relato de dificuldade que a mesma tenha omitido e que a enfermagem não tenha observado. Para Oliveira MJM e Dunningham WA (2015), comentam que o impacto da depressão no RN vai depender de como está afetando o comportamento, a cognição e as emoções da própria mãe. Neste contexto, a perspectiva sobre a depressão afeta o RN pela modificação dos modelos de interação mãe-filho ou pelo aumento da discórdia entre o casal, que tem efeitos negativos no desenvolvimento infantil. 
De acordo com Mozzaquatro O e Arpini DM (2017), a interrupção do sono está correlacionada com os sinais depressivos no pós-parto. Essa fragmentação do sono interfere negativamente no bem-estar da mulher e do RN, na maternidade e na amamentação, assim como na saúde e desenvolvimento do mesmo. O sono adequado no pós-parto beneficia a saúde da puérpera e a sua capacidade de cuidar do seu bebê.

Com à ansiedade/perda da segurança, a puérpera pode desenvolver uma preocupação excessiva quanto à saúde e ao desenvolvimento do RN, de modo que essa puérpera venha se preocupar menos e se sentir incapaz e a mesma estará mais suscetível à elevação do seu quadro depressivo. A depressão e a ansiedade materna refletem negativamente no desenvolvimento do bebê e são capazes de acarretar danos nas relações pessoais que esta possa estabelecer ao longo da sua vida (MELO SB, et al., 2018).

\section{CONSIDERAÇÕES FINAIS}

Foi possível verificar como os impactos do baby blues podem influenciar durante a gestação e no pósparto. As habilidades relacionadas à realização dos cuidados primários foram elevadas e, no geral, positivas. Admite-se que, como são atitudes relativas às práticas de cuidados básicos relacionados à sobrevivência, saúde e higiene, esse fato pode estar interligado ou condicionado a possibilidade as mães contarem com uma rede de apoio que as auxilie nesses cuidados incluídos os seus familiares.

\section{REFERÊNCIAS}

1. ANDRADE M, et al. Tristeza materna em puérperas e fatores associados. Revista Portuguesa de Enfermagem de Saúde Mental, 2017; (18): 8-13.

2. ARRAIS AR, ARAUJO TCCF. Depressão pós-parto: uma revisão sobre fatores de risco e de proteção. Psicologia, Saúde \& Doenças, 2017; 18 (3): 828-845.

3. BISSOLI MF. Development of Children's Personality: The role of early childhood education. Psicologia em Estudo, 2014; 19 (4): 587-597.

4. BRASIL. Ministério da Saúde. Conselho Nacional de Saúde. Resolução 466/2012. Aprova as seguintes Normas para Pesquisas Envolvendo Seres Humanos. 2012a.

5. CARDILLO VA, et al. Identificação de sintomas depressivos no período pós-parto em mães adolescentes. Rev Eletr Enf, 2016; 18 (1): 1149-24.

6. COSTA TE, et al. Depressão pós-parto: conhecimento sobre os sinais e sintomas em puérperas. Revista de Ciências da Saúde Nova Esperança, 2016; 14(1): 59-66.

7. FONSECA A, CANAVARRO MC. Depressão Pós-Parto. PROPSICO: Programa de atualização em Picologia Clínica e da Saúde-Ciclo 1, 2017; 111-164.

8. FRANCES A. Fundamentos do Diagnóstico Psiquiátrico: Respondendo às Mudanças do DSM-5. Porto Alegre: Artmed, 2015; 51p.

9. FREITAS DR, et al. Alojamento conjunto em um hospital universitário: depressão pós-parto na perspectiva do enfermeiro. Revista de pesquisa cuidado é fundamental online, 2014; 6(3): 1202-1211.

10. GONÇALVES AMC, et al. Prevalence of depression and associated factors in women covered by Family Health Strategy. Jornal Brasileiro de Psiquiatria, 2018; 67(2): 101-109.

11. GREINERT BRM, MILANI RG. Depressão pós-parto: uma compreensão psicossocial. Psicologia: teoria e prática, $2015 ; 17(1): 26-36$.

12. GUIMARÃES RM, et al. Factors associated to the type of childbirth in public and private hospitals in Brazil. Revista Brasileira de Saude Materno Infantil, 2017; 17(3): 571-580.

13. HARTMANN JM et al. Depressão entre puérperas: prevalência e fatores associados. Cadernos de Saúde Pública, 2017; 33: e00094016.

14. LIMA MOP, et al. Sintomas depressivos na gestação e fatores associados: estudo longitudinal. Acta Paulista de Enfermagem, 2017; 30(1): 39-46.

15. LIMA SS, et al. Depressão Pós-Parto: Um Olhar Criterioso Da Equipe De Enfermagem. Caderno de GraduaçãoCiências Biológicas e da Saúde-UNIT, 2018; 4(3): 71.

16. MARTINIANO CS, et al. Legalização da prescrição de medicamentos pelo Enfermeiro no Brasil: história, tendências e desafios. Texto \& Contexto-Enfermagem, 2015; 24(3): 809-817.

17. MARCONI, MA; LAKATOS, EV. Técnicas de pesquisa: planejamento e execução de pesquisas, amostragens e técnicas de pesquisa, elaboração, análise e interpretação de dados. 8 ed. São Paulo: Atlas, 2017; 328p.

18. MARTINEZ P, et al. Barreiras de acesso a tratamento para mães como depressão pós-parto em centros de atenção primária: um modelo preditivo. Revista Latino Americana de Enfermagem. 2016; 24.

19. MELO SB, et al. Sintomas depressivos em puérperas atendidas em unidades de saúde da família. Revista Brasileira de Saúde Materno Infantil, 2018; 18(1): 163-169.

20. MEIRA BM, et al. Challenges for primary healthcare professionals in caring for women with postpartum depression. Texto \& Contexto-Enfermagem, 2015; 24(3): 706-712. 
21. MORAES GPA, et al. Triagem e diagnóstico da depressão pós-parto: quando e como? Tendências em psiquiatria e psicoterapia, 2017; 39(1): 54-61.

22. MORAIS MLS, et al. Fatores psicossociais e sociodemográficos associados à depressão pós-parto: Um estudo em hospitais público e privado da cidade de São Paulo, Brasil. Estudos de psicologia (Natal), 2015; 20(1): 40-49.

23. MOZZAQUATRO CO, ARPINI DM. Planejamento familiar e papéis parentais: o tradicional, a mudança e os novos desafios. Psicologia: Ciência e Profissão, 2017; 37(4): 923-938.

24. NARDI CGA, et al. Bebês com Sequência de Pierre Robin: saúde mental materna e interação mãe-bebê. Estudos de Psicologia (Campinas), 2015; 32(1): 129-140.

25. MÜLLER PW, et al. A relação mãe-bebê na presença e na ausência de sintoma psico funcional no bebê: um estudo comparativo. Boletim Academia Paulista de Psicologia, 2017; 37(93): 229-251.

26. OLIVEIRA CC, et al. Depressão Pós-Parto. Revista Intercâmbio, 2019; 15: 147-158.

27. OLIVEIRA MJM, DUNNINGHAM WA. Prevalência e fatores de risco relacionados a depressão pós-parto em Salvador. Revista Brasileira de Neurologia e Psiquiatria, 2015; 19(2).

28. PESSOA, L. Assistência de Enfermagem à puérpera com depressão pós-parto no ambiente hospitalar. (Trabalho de Conclusão de Curso Graduação). Universidade do Estado do Amazonas, Manaus, 2014; 16p.

29. RAMOS ASMB, et al. Fatores associados à depressão pós-parto: Revisão Integrativa. Enciclopédia Biosfera. Centro Científico Conhecer, Goiânia, 2018; 15(27): 4-6.

30. RIBEIRO DG, et al. Saúde mental, interação mãe-criança e desenvolvimento ao final do primeiro ano de vida. Paidéia (Ribeirão Preto), 2014; 24(59): 331-339.

31. SANTOS JMM, ALMEIDA AB. The Understanding Of The Nurse Facing The Identification Of Postpartum Depression. Simpósio de TCC e Seminário de IC, 2016; 2: 1364.

32. SILVA TR, GONTIJO CS. A Família e o Desenvolvimento Infantil sob a Ótica da Gestalt-Terapia. IGT na Rede, 2016; 13(24): 15-36. 